\title{
Differences in glucose control in type 1 diabetes mellitus after bicycling and after yoga
}

\section{ABSTRACT}

Physical activity (PA) is an important recommendation for type 1 diabetes mellitus (T1DM) patients. However, it is commonly thought that higher intensities result in elevating the risk of hypoglycemia immediately after physical activity. We present a case report of a diabetes patient whose blood glucose levels dropped more immediately after low-level aerobic intensity yoga exercises compared with the level drop immediately after high-level aerobic intensity bicycling exercises. The results suggest that due to the difficulty in predicting the glycemic response to physical exercise it is important to better prepare T1DM patients when prescribing them with PA. (Clin Diabet 2020; 9; 5: 335-337)

Key words: diabetes mellitus type 1, bicycling, yoga, glucose, hypoglycemia

\section{Introduction}

Physical activity (PA) is known to improve insulin sensitivity [1], help in body-mass maintenance, reduce the occurrence of hyperglycemia [2] and to revert

\section{Address for correspondence:}

Eyal Weissblueth

Ohalo Academic College

School of Physical Education

Qatzrin, 1290000, Israel

Phone: +972-54-4376868

Fax: +972-4-6825011

e-mail: eyalw@ohalo.ac.il

Clinical Diabetology 2020, 9; 5: 335-337

DOI: $10.5603 /$ DK.2020.0040

Received: 12.07 .2020

Accepted: 12.09 .2020 diabetic condition into a non-diabetic state [3]. Nevertheless, exercise too often results in hypoglycemia in people with T1DM. Assessment of the relative intensity during the effort, resulting in the consumption of the metabolic raw material glucose or free fatty acids, is usually based on the relative heart rate (HR) as a percentage of age-predicted maximal HR. Various yoga exercises can be used as a complementary or integrative therapy for T1DM [4] but quantifying and characterizing the various yoga exercises is difficult because of differences in practice time and relative strength of various yoga positions.

We have recently encountered a patient who practiced exertion training in the form of bicycling as well as yoga sessions. She experienced episodes of hypoglycemia following yoga sessions, unlike after bicycling. We, therefore, analyzed simultaneous recordings of her cardiac activity with glycemic recordings. Our findings raise a few questions regarding the prescription of varying activities to people with T1DM.

\section{Case report}

$A A$, a 58 years old woman with 30-years of T1DM had been treated by a continuous insulin pump (Insulet Omnipod ENT450) and monitored by a continuous glucose monitor that was not synchronous with the pump. Her daily dose of insulin was 20 units in the form of basal infusion. Her glycaemia target was $100 \mathrm{mg} / \mathrm{dL}$, 1 unit of insulin per $15 \mathrm{~g}$ of carbohydrates in all meals, and sensitivity $55 \mathrm{mg} / \mathrm{dL}$ (1 unit of insulin decreased glycemia by $55 \mathrm{mg} / \mathrm{dL}$ for the same whole day). Her $\mathrm{HbA}_{1 \mathrm{c}}$ at the analysis time was $5.8 \%$. Her body mass index (BMI) was 21.7 and she practiced PA four times 
a week for many years, which more recently included yoga. Using Garmin 395LM to monitor HR beats per minute (BPM) we downloaded the recordings of 11 sessions of bicycling and 11 sessions of yoga from her continuous glucose monitor, each session lasted 60 minutes during 40 days. The sessions of bicycling were done twice a week at 06:30 in the morning before breakfast whereas the yoga sessions were done twice a week at 19:00 in the evening 4 hours after a meal. Cycling was done on an Argon-18 bicycle connected to Wahoo Kickr ANT+ smart computerized trainer with personal adjusted wattage. The intensity of her cardiac response to training was calculated in reference to a peak HR of 175 BPM during exertion training. During bicycling her $\mathrm{HR}$ reached an average of $146 \pm 6 \mathrm{BPM}$ for the whole period ( $84 \%$ maximal HR), whereas during the yoga session it was $120 \pm 9$ BPM ( $68 \%$ maximal HR). Insulin adjustments of 0.5 units each were done twice during cycling and three times during yoga. This study was approved in advance by the Institutional Review Board of the College. AA voluntarily provided written informed consent before participating.

Interestingly, her glucose levels before bicycling were $94 \pm 11 \mathrm{mg} / \mathrm{dL}$ and rose to $145 \pm 11 \mathrm{mg} / \mathrm{dL}$ after the conclusion of each session $(t(10)=-9.27$, $P<0.001, d=-2.79)$, whereas mean glucose levels before yoga was $153 \pm 25 \mathrm{mg} / \mathrm{dL}$ and dropped to $69 \pm$ $\pm 10 \mathrm{mg} / \mathrm{dL}(\mathrm{t}(10)=7.00, \mathrm{P}<0.001, \mathrm{~d}=1.94)$, so was the difference in glucose level between activities $(P<0.01)$. It is important to clarify here that neither before bicycling nor before yoga did she eat any significant amount of food.

Plotting individual events' glucose level posttraining against mean HR during the sessions in both yoga and bicycling showed no trend. We found no correlations neither before nor after training, between the increment of glucose levels and that of mean HR for bicycling or for yoga training $(r(11)=-0.069$, $\mathrm{P}=0.84 ; \mathrm{r}(11)=-0.122, \mathrm{P}=0.69$ respectively).

\section{Discussion}

The main aim of this study was to assess the effect of PA intensity on blood glucose concentration during and immediately after planned and controlled physical training. However, while previous studies [5] examined the difference in hormonal response to various efforts in the same activity (bicycling), this study compared two different planned PA aerobic/dynamic (bicycling) and aerobic/anaerobic and partly static (yoga) on the same person in a real-life situation.

The results of this study, which relied on HR as an assessment of the intensity of PA suggested that intensity of $82 \%$ of maximum HR (bicycle) reduced the risk of hypoglycemia immediately after exercise compared with the intensity of $68 \%$ (yoga) similar to earlier studies $[5,6]$. The inverse $U$ theory [5] seems to explain the change not only in the concentration of blood glucose but also its direction a high excretion rate (Ra) at high PA intensity versus a higher increase in the rate of utilization ( $\mathrm{Rd}$ ) at a moderate PA intensity [5]. The catecholamines' response to the relatively high PA intensity is likely to cause Ra level to exceed that of Rd [7], while light and moderate intensities of PA require a steady increase of glucose to maintain euglycemia [5] so that the "Dose-Response" effect was reflected in this case.

The repertoire of responses that are aimed at preventing hypoglycemia includes withholding insulin release, secretion of glucagon, secretion of catecholamines, growth hormone, and cortisol. It is not clear how the ability to respond with glucagon is compromised in T1DM patients and several possible explanations were given. Indeed, in senior T1DM patients, the response to hypoglycemia is so compromised that epinephrine secretion becomes the main protection line against events of hypoglycemia [7]. The response to yoga sessions by our patient appeared to be paradoxical since no major exertion necessitating glucose uptake was recorded (non-elevated HR). It is of note that in mice, passive stretching induces GLUT-4 translocation from an intracellular membrane-rich fraction to a plasma membrane-rich fraction and accelerates glucose uptake in hind-limb muscles [8]. Most likely in our patient, yoga did not evoke adrenergic response while increasing glucose uptake consequent to muscle stretching.

\section{Conclusions}

This fundamental difference in blood glucose response between aerobic exertion and yoga training should become a major point of attention for T1DM patients, since the percentage of the peak HR is perhaps not sufficiently sensitive among those who engage in a variety of PAs and are concerned about a hypoglycemic event due to exercise, especially in those where the anaerobic component is not clear. While keeping in mind that different training activities produce different blood glucose responses it would be appropriate to arrange a "personal suit" for the diabetic trainee. This should include some pre or post-exercise readily absorbable glucose supplement as well as calibration of insulin according to the intensity of the planned effort and limits of the intensity of the training so that the fear of a hypoglycemic event at or shortly thereafter will decrease.

Because with mild/moderate cardiovascular exertion, a stronger decrease in blood glucose was observed 
than with higher intensity exertion, before a yoga workout our patient should reduce administration of insulin or eat a carbohydrate dose before the workout. During cardiovascular cycling training, where there was no observed strong decrease in glucose concentration there is no need to adjust insulin administration because during the exertion glycogen stores release glucose at a level appropriate to the required effort and the insulin administered.

\section{Conflict of interest}

All authors declared to have no conflict of interest that is relevant to the subject matter or materials included in this work.

\section{REFERENCES}

1. Yaribeygi H, Atkin SL, Simental-Mendía LE, et al. Molecular mechanisms by which aerobic exercise induces insulin sensitivity. J Cell Physiol. 2019; 234(8): 12385-12392, doi: 10.1002/jcp.28066, indexed in Pubmed: 30605232.

2. Zhao F, Wu W, Feng $X$, et al. Physical activity levels and diabetes prevalence in US adults: findings from NHANES 2015-2016.
Diabetes Ther. 2020; 11(6): 1303-1316, doi: 10.1007/s13300020-00817-x, indexed in Pubmed: 32323158.

3. Lean M, Leslie W, Barnes A, et al. Primary care-led weight management for remission of type 2 diabetes (DiRECT): an open-label cluster-randomised trial. The Lancet. 2018; 391(10120): 541-551, doi: 10.1016/s0140-6736(17)33102-1.

4. Thangasami $S$, Chandani $A$. Emphasis of yoga in the management of diabetes. Journal of Diabetes \& Metabolism. 2015; 6(10), doi: 10.4172/2155-6156.1000613.

5. Shetty VB, Fournier PA, Davey RJ, et al. Effect of exercise intensity on glucose requirements to maintain euglycemia during exercise in type 1 diabetes. J Clin Endocrinol Metab. 2016; 101(3): 972 980, doi: 10.1210/jc.2015-4026, indexed in Pubmed: 26765581.

6. Mitranun W, Deerochanawong C, Tanaka H, et al. Continuous vs interval training on glycemic control and macro- and microvascular reactivity in type 2 diabetic patients. Scand J Med Sci Sports. 2014; 24(2): e69-e76, doi: 10.1111/sms.12112, indexed in Pubmed: 24102912

7. Moser O, Tschakert G, Mueller A, et al. Atypical blood glucose response to continuous and interval exercise in a person with type 1 diabetes: a case report. J Med Case Rep. 2017; 11(1): 176, doi: 10.1186/s13256-017-1355-7, indexed in Pubmed: 28662684.

8. Sato K, Nishijima T, Yokokawa T, et al. Acute bout of exercise induced prolonged muscle glucose transporter- 4 translocation and delayed counter-regulatory hormone response in type 1 diabetes. PLoS One. 2017; 12(6): e0178505, doi: 10.1371/journal. pone.0178505, indexed in Pubmed: 28570686. 